\title{
Effect of Sending Educational Video Clips via Smartphone Mobile Messenger on Bowel Preparation before Colonoscopy
}

\author{
Sung Chan Jeon*, Jae Hyun Kim, Sun Jung Kim, Hye Jung Kwon, Youn Jung Choi, Kyoungwon Jung, Sung Eun Kim, Won Moon, \\ Moo In Park and Seun Ja Park
}

Department of Gastroenterology, Kosin University College of Medicine, Busan, Korea

Background/Aim: We aimed to evaluate the efficacy of sending educational video clips via smartphone mobile messenger (SMM) on enhancing bowel preparation before colonoscopy.

Methods: This was a prospective, endoscopist-blinded, randomized controlled study. Patients in the SMM group received two video clips sent via SMM that explained the diet and regimen for bowel preparation, whereas those in the control group did not receive any video clips. We compared the quality of bowel preparation between the two groups, which was assessed by an endoscopist using the Ottawa scale.

Results: Between August and November 2014, 140 patients in the SMM group and 141 patients in the control group underwent colonoscopic examination. The total Ottawa score of the SMM group was significantly lower than that of the control group $(5.47 \pm 1.74$ vs. $5.97 \pm 1.78, p=0.018$ ). These results were particularly prominent in the younger age group; the total Ottawa score of patents in the SMM group aged $<40$ years was significantly lower than that of patients in the control group aged $<40$ years $(5.10 \pm 1.55 \mathrm{vs.} 6.22 \pm 2.33$, $p=0.034$ ).

Conclusions: We demonstrated that sending educational video clips via SMM could result in better bowel preparation, especially in the younger age group. Clin Endosc 2019;52:53-58

Key Words: Colonoscopy; Bowel preparation; Education

\section{INTRODUCTION}

Colonoscopy is an important tool in the diagnosis and treatment of colorectal disease, and it should be preceded by adequate pretreatment in order to facilitate safe and effective testing. ${ }^{1}$ However, bowel preparation is inadequate for up to one-third of all colonoscopies in daily clinical practice. . $^{2-4}$

Received: April 26, 2018 Revised: June 1, 2018

Accepted: June 2, 2018

Correspondence: Seun Ja Park

Department of Gastroenterology, Kosin University College of Medicine, 262 Gamcheon-ro, Seo-gu, Busan 49267, Korea

Tel: +82-51-990-5061, Fax: +82-51-990-5055, E-mail: parksj6406@daum.net ORCID: https://orcid.org/0000-0003-3217-5115

*Sung Chan Jeon and Jae Hyun Kim contributed equally to this article.

(cc) This is an Open Access article distributed under the terms of the Creative Commons Attribution Non-Commercial License (http://creativecommons.org/ licenses/by-nc/3.0) which permits unrestricted non-commercial use, distribution, and reproduction in any medium, provided the original work is properly cited.
Inadequate bowel preparation can increase the risk of missed lesions, risk of complications following the procedure, procedure time, and need to re-perform colonoscopic examination. ${ }^{5}$ Adequate bowel preparation is closely linked to patient compliance, such as whether the patient used the appropriate administration method or had a low-residue diet. ${ }^{2}$ To improve patient compliance, it may be helpful to enhance patient education on the importance of colonic cleansing before colonoscopy. Recently, several studies have reinforced the need for patient education on colonic cleansing prior to colonoscopy using a variety of methods, including cartoons, video clips, intensive education, or telephone-based re-education. ${ }^{6-9}$ These studies showed that such educational methods may increase the quality of bowel preparation from $11.3 \%$ to $32.6 \%$; however, many patients still do not achieve adequate bowel preparation. Therefore, further studies are needed to increase the effectiveness of patients' pre-colonoscopy colonic cleansing.

Since the launch of the iPhone (Apple Computer Inc., 
Cupertino, CA, USA) in 2007, smartphone usage has risen remarkably worldwide. In Korea, the smartphone penetration rate is about $80 \%$, and mobile messenger usage among smartphone users is up to $90 \%$. Among various mobile messenger applications, KakaoTalk (Kakao Co., Seoul, Korea) is used the most in Korea and has the advantage of easily transferring pictures and videos via messenger. In this study, we hypothesized that sending educational video clips via smartphone mobile messenger (SMM, KakaoTalk) could be a useful tool to improve the quality of colonic cleansing before colonoscopy owing to the ease of transferring photos and videos to others and the capacity to view them repeatedly at any time. We aimed to evaluate the efficacy of sending educational video clips on colonic cleansing before colonoscopy via SMM and to assess whether it could be applied in clinical practice.

\section{MATERIALS AND METHODS}

\section{Patients}

This is a prospective, endoscopist-blinded, randomized controlled study. The study protocol was approved by the Institutional Review Board of Kosin University Gospel Hospital (KUGH 2014-07-074). Patients aged >30 years who were undergoing screening colonoscopy at the health examination center of Kosin University Gospel Hospital in Korea were enrolled. Patients with a history of abdominal surgery, inflammatory bowel disease, chronic kidney disease, heart failure, ascites, ileus or intestinal obstruction, or allergy to bowel preparation drugs and those who were pregnant were excluded. Written informed consent was obtained from all participants before enrollment in this study. All patients listened to explanations about the study purpose given by a well-trained nurse in the health examination center. The nurse provided education with an informational brochure on colonoscopy, which included the diet schedule (low-fiber diet for three days and soft diet for dinner the day before the colonoscopy), administration method of the bowel preparation regimen, process of colonoscopic examination, and side effects of the regimen. Then, subjects were randomized to either the SMM or control group using the table of random numbers. Patients in the SMM group received two video clips sent via KakaoTalk, whereas those in the control group did not receive any video clips.

\section{Creation of the video clips}

A study investigator (SCJ) created two video clips introducing the diet and regimen for colonic cleansing before colonoscopy. The first video clip introduced the diet for colonic cleansing before colonoscopy. In this video clip, a low-fiber diet for three days and soft diet for dinner the day before the colonoscopy were recommended, and foods to avoid including watermelon, oriental melon, grape, nut products, kimchi, vegetables, and seaweed were emphasized. The second video clip introduced the regimen for bowel preparation. This video clip explained the detailed preparation method of the polyethylene glycol electrolyte powder (PEG-ELP), which involved drinking $500 \mathrm{~mL}$ water after consuming 1 L PEG-ELP between 9:00 and 11:00 p.m. on the day before colonoscopy and drinking an additional $500 \mathrm{~mL}$ water after consuming $1 \mathrm{~L}$ PEG-ELP between 5:00 and 7:00 a.m. on the day of the colonoscopy. Each video clip was approximately 2-min long.

\section{Data collection and colonoscopy}

On the day of the colonoscopy, all patients were interviewed by a study investigator (SCJ), and they filled out a questionnaire on aspects such as the viewing of each video, types of food consumed, start time of initial bowel preparation, time of final bowel preparation, and last stool color. Patient compliance was assessed as good or bad based on the food types consumed and time of bowel preparation. Side effects were categorized as mild, moderate, or severe. Colonoscopic examinations were performed by one experienced endoscopist (SJK) who was blinded to the group information. All colonoscopic examinations were performed between 09:00 a.m. to 01:00 p.m. During the examination, insertion time, withdrawal time, polyp detection, and bowel preparation scale were recorded. The bowel preparation scale was assessed according to the Ottawa bowel preparation scale, ${ }^{10}$ with a score of $0-4$ for the rectosigmoid, middle, and right colon and 0-2 for quantity of residual fluid. The total score was calculated by summing all scores. The scale ranges from 0 (perfect) to 14 (inadequate). When a polyp was detected during colonoscopic examination, biopsy and histologic assessments were performed to identify the adenoma component. Then, the polyp detection rate (PDR) (the number of colonoscopies in which one or more polyps were detected divided by the total number of colonoscopies) and adenoma detection rate (ADR) (the number of colonoscopies in which one or more adenomas were detected divided by the total number of colonoscopies) were assessed.

\section{Endpoints}

The primary endpoint in this study was the quality of the bowel preparation, which was assessed by the endoscopist using the Ottawa scale. Adequate bowel preparation was defined as an Ottawa score of $<6$ at the time of colonoscopy. Secondary endpoints were colonoscopic insertion and withdrawal time, PDR, ADR, side effects during bowel preparation, and rate of sleep disturbance due to bowel preparation. Severe side effects were defined as $\geq 10$ nausea/vomiting episodes or intol- 
erable abdominal pain/discomfort.

\section{Statistical analysis}

The number of patients in each group was calculated with an alpha of 0.05 and a power of 0.8 , assuming a $12 \%$ difference in the rate of colonic cleansing based on previous studies. Accounting for a dropout rate of $10 \%$, at least 153 subjects in each group were needed for the study. Continuous data with normal distributions are expressed as mean \pm standard deviation, and categorical data are presented as the number of subjects (\%). Student's $t$-test and the chi-square test were performed for continuous and categorical variables, as appropriate. To analyze the factors associated with adequate bowel preparation, univariate and multivariate logistic regression analyses were performed. $P$-values of $<0.05$ were considered statistically significant. Statistical analyses were performed using SPSS version 23.0 (IBM Co., Armonk, NY, USA).

\section{RESULTS}

\section{Patient characteristics}

Between August and November 2014, 306 patients signed written informed consent forms and were randomized to the SMM and control groups. After randomization, 25 patients cancelled appointments and did not undergo colonoscopy. Finally, 140 patients from the SMM group and 141 patients from the control group underwent colonoscopic examination. The patient selection flowchart is presented in Fig. 1. Baseline characteristics are summarized in Table 1. The mean age of all participants was $48.3 \pm 9.9$ years, and 161 (57.3\%) patients were men. Patients in the SMM group were significantly younger and had lower body mass index (BMI). All participants performed bowel preparation with PEG-ELP.

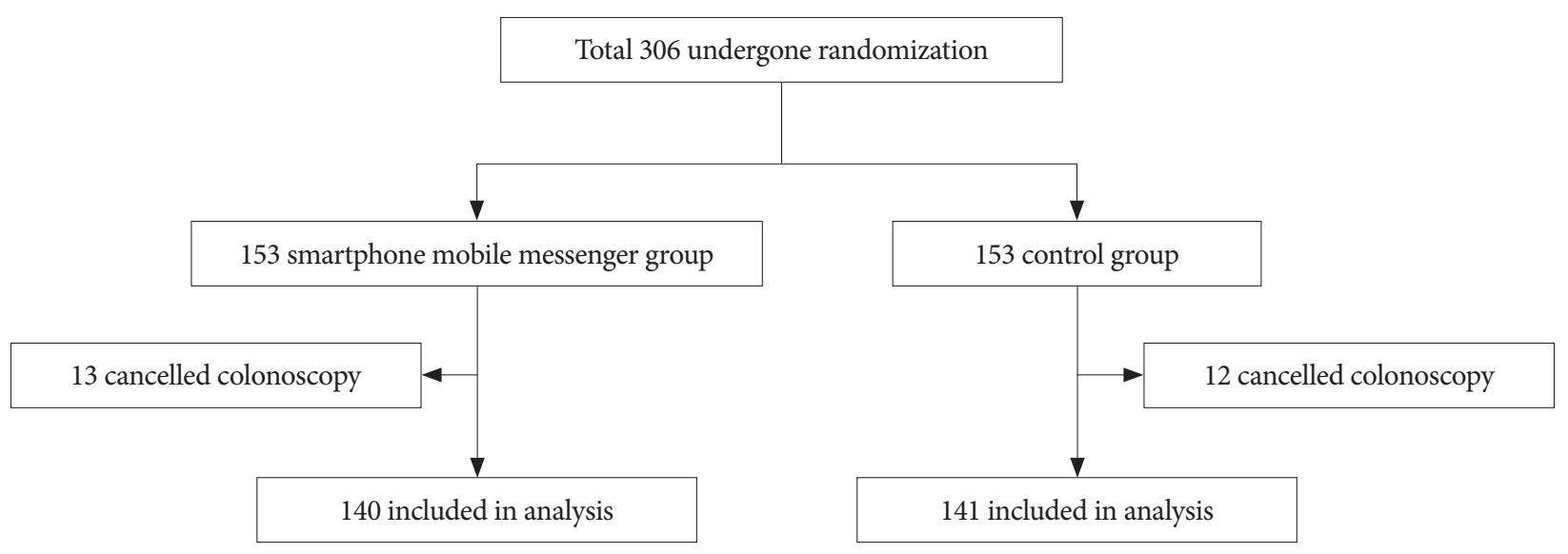

Fig. 1. Flowchart of the study.

Table 1. Baseline Characteristics

\begin{tabular}{|c|c|c|c|}
\hline & SMM group $(n=140)$ & Control group $(n=141)$ & $p$-value \\
\hline Age (yr) & $46.7 \pm 9.9$ & $49.9 \pm 9.6$ & 0.006 \\
\hline Sex & & & 0.959 \\
\hline Male & $80(57.1)$ & $81(57.4)$ & \\
\hline Female & $60(42.9)$ & $60(42.6)$ & \\
\hline BMI $\left(\mathrm{kg} / \mathrm{m}^{2}\right)$ & $23.5 \pm 3.0$ & $24.5 \pm 3.1$ & 0.009 \\
\hline Previous experience of colonoscopy & & & 0.257 \\
\hline No & $67(47.9)$ & $58(41.1)$ & \\
\hline Yes & $73(52.1)$ & $83(58.9)$ & \\
\hline \multicolumn{4}{|l|}{ Bowel preparation type } \\
\hline PEG-ELP & 140 & 141 & 1.000 \\
\hline Others & 0 & 0 & \\
\hline
\end{tabular}

Values are presented as mean \pm standard deviation or $n(\%)$.

SMM, smartphone mobile messenger; BMI, body mass index; PEG-ELP, polyethylene glycol electrolyte powder. 


\section{Endpoints}

As shown in Table 2, there were no significant differences between the two groups in the assessment of patient compliance, status of last defecation after bowel preparation, and adequate bowel preparation according to the Ottawa score $(<6)$. In the SMM group, the odds ratio predicting adequate bowel preparation (Ottawa score $<6$ ) was 1.39 (95\% confidence interval, $0.87-2.22 ; p=0.171)$. The result did not change appreciably after adjusting for age, sex, BMI, and previous experience of colonoscopy. However, the total Ottawa score was significantly lower in the SMM group than in the control group (5.47 \pm 1.74 vs. $5.97 \pm 1.78, p=0.018)$. In particular, the Ottawa scores for the right and middle colon were significantly lower in the SMM group than in the control group (right colon, $1.99 \pm 0.59$ vs. $2.12 \pm 0.52, p=0.035$; middle colon, $1.48 \pm 0.67$ vs. $1.67 \pm 0.64, p=0.013)$. These results were particularly prominent in the younger age group; the total Ottawa score of patients in the SMM group aged $<40$ years was significantly lower than that of patients in the control group aged $<40$ years $(5.10 \pm 1.55$ vs. $6.22 \pm 2.33, p=0.034$; Table 3 ). However, these differences between the two groups were not significant in patients aged $>40$ years. Under 40 years of age, the patient compliance was significantly better in the SMM group than in the control group (data not shown). Between the two groups, the differences in cecal intubation time, severe symptoms during bowel preparation, and sleep disturbances were not significant, whereas the withdrawal time was longer in the control group than in the SMM group, and PDR and ADR were higher in the control group than in the SMM group (Table 4).

\section{DISCUSSION}

In this prospective, endoscopist-blinded, randomized controlled study, we evaluated the efficacy of sending educational video clips via SMM on facilitating bowel preparation. Upon analyzing the results, we found that sending educational video clips via SMM may result in better bowel preparation, especially in the right and middle colon. In addition, we identified that this educational method could be especially helpful to patients in the younger age group.

Adequate bowel preparation is an important factor for achieving an efficient colonoscopic examination. The quality of bowel preparation is associated with both the cecal intubation rate and PDR, which are key quality indicators in colonoscopy, ${ }^{10,11}$ whereas inadequate bowel preparation can result in an increased number of missed lesions and complication rates, as well as unnecessary costs when the examination has to be re-scheduled. ${ }^{12,13}$ Several studies on bowel preparation education before colonoscopy have recently been reported, and they indicated that educating patients with various methods could effectively improve bowel preparation for colonoscopy ${ }^{6,8,14-17}$ In our study, we investigated whether sending educational video clips on bowel preparation before colonoscopy via SMM, an easy way to send photos and videos to participants that is used by most people in Korea, enhances the quality of pre-colonoscopy bowel preparation.

We defined the cut-off for adequate bowel preparation as an Ottawa score of 6 or less and evaluated the difference between the SMM and control groups in the assessment of adequate bowel preparation according to the Ottawa score $(<6)$. The percentage of patients with an Ottawa score of $<6$ was high-

Table 2. Bowel Preparation Outcomes

\begin{tabular}{|c|c|c|c|}
\hline & SMM group $(n=140)$ & Control group $(n=141)$ & $p$-value \\
\hline Compliance & & & 0.069 \\
\hline Good & $136(97.1)$ & $129(91.5)$ & \\
\hline $\mathrm{Bad}$ & $4(2.9)$ & $12(8.5)$ & \\
\hline Last defecation after bowel preparation & & & 0.121 \\
\hline Clear, no residue & $30(21.4)$ & $20(14.2)$ & \\
\hline Yellow residue & $110(78.6)$ & $121(85.8)$ & \\
\hline Ottawa score & $5.47 \pm 1.74$ & $5.97 \pm 1.78$ & 0.018 \\
\hline Right & $1.99 \pm 0.59$ & $2.13 \pm 0.52$ & 0.035 \\
\hline Middle & $1.48 \pm 0.67$ & $1.67 \pm 0.64$ & 0.013 \\
\hline Rectosigmoid & $1.23 \pm 0.55$ & $1.27 \pm 0.56$ & 0.538 \\
\hline Fluid & $0.77 \pm 0.63$ & $0.89 \pm 0.66$ & 0.113 \\
\hline Adequate bowel preparation for colonoscopy (Ottawa score $<6$ ) & $71(50.7)$ & $60(42.6)$ & 0.170 \\
\hline
\end{tabular}

Values are presented as mean \pm standard deviation or $n(\%)$.

SMM, smartphone mobile messenger. 
Table 3. Bowel Preparation Outcomes according to Age Group

\begin{tabular}{|c|c|c|c|}
\hline & SMM group $(n=140)$ & Control group $(n=141)$ & $p$-value \\
\hline Under 40 yr $(n=66)$ & $(n=39)$ & $(n=27)$ & \\
\hline Ottawa score & $5.10 \pm 1.55$ & $6.22 \pm 2.33$ & 0.034 \\
\hline Right & $1.97 \pm 0.54$ & $2.19 \pm 0.62$ & 0.147 \\
\hline Middle & $1.46 \pm 0.64$ & $1.70 \pm 0.78$ & 0.172 \\
\hline Rectosigmoid & $1.08 \pm 0.48$ & $1.37 \pm 0.74$ & 0.078 \\
\hline Fluid & $0.59 \pm 0.59$ & $0.96 \pm 0.65$ & 0.019 \\
\hline $41-50$ yr $(n=90)$ & $(n=51)$ & $(n=39)$ & \\
\hline Ottawa score & $5.59 \pm 1.76$ & $5.82 \pm 1.60$ & 0.521 \\
\hline Right & $1.98 \pm 0.65$ & $2.13 \pm 0.52$ & 0.247 \\
\hline Middle & $1.49 \pm 0.64$ & $1.72 \pm 0.61$ & 0.091 \\
\hline Rectosigmoid & $1.27 \pm 0.57$ & $1.21 \pm 0.41$ & 0.503 \\
\hline Fluid & $0.84 \pm 0.61$ & $0.77 \pm 0.67$ & 0.587 \\
\hline $51-60$ yr $(n=98)$ & $(n=38)$ & $(n=60)$ & \\
\hline Ottawa score & $5.50 \pm 1.97$ & $5.73 \pm 1.61$ & 0.524 \\
\hline Right & $1.95 \pm 0.61$ & $2.08 \pm 0.50$ & 0.232 \\
\hline Middle & $1.39 \pm 0.76$ & $1.58 \pm 0.62$ & 0.181 \\
\hline Rectosigmoid & $1.26 \pm 0.60$ & $1.23 \pm 0.56$ & 0.804 \\
\hline Fluid & $0.89 \pm 0.69$ & $0.83 \pm 0.59$ & 0.638 \\
\hline Over 61 yr $(n=27)$ & $(n=12)$ & $(n=15)$ & \\
\hline Ottawa score & $6.08 \pm 1.31$ & $6.87 \pm 1.55$ & 0.176 \\
\hline Right & $2.25 \pm 0.45$ & $2.27 \pm 0.46$ & 0.925 \\
\hline Middle & $1.75 \pm 0.62$ & $1.87 \pm 0.52$ & 0.599 \\
\hline Rectosigmoid & $1.42 \pm 0.52$ & $1.40 \pm 0.51$ & 0.933 \\
\hline Fluid & $0.67 \pm 0.49$ & $1.33 \pm 0.82$ & 0.015 \\
\hline
\end{tabular}

Values are presented as mean \pm standard deviation.

SMM, smartphone mobile messenger.

Table 4. Colonoscopy Outcomes and Side Effects

\begin{tabular}{lccc}
\hline & SMM group $(\boldsymbol{n = 1 4 0 )}$ & Control group $(\boldsymbol{n}=\mathbf{1 4 1})$ & $\boldsymbol{p}$-value \\
\hline Cecal intubation time (min) & $2.7 \pm 1.6$ & $2.5 \pm 1.3$ & 0.352 \\
Withdrawal time (min) & $10.2 \pm 2.3$ & $10.9 \pm 2.2$ & 0.007 \\
Polyp detection & $62(44.3)$ & $84(59.6)$ & 0.010 \\
Adenoma detection & $32(22.9)$ & $49(34.8)$ & 0.028 \\
Severe side effects during bowel preparation & & & 0.377 \\
Nausea/vomiting & $4(2.8)$ & $8(5.7)$ & 1.000 \\
Abdominal pain/discomfort & $8(5.7)$ & $8(5.7)$ & 0.131 \\
Sleep disturbance & $11(7.9)$ & $5(3.5)$ & \\
\hline
\end{tabular}

Values are presented as mean \pm standard deviation or $n(\%)$.

SMM, smartphone mobile messenger.

er in the SMM group than in the control group (50.7\% vs. $42.6 \%$ ), although the difference was not statistically significant. In comparison to the total Ottawa score, bowel preparation was significantly better in the SMM group than in the control group. These results suggest that sending educational video clips via SMM may be a helpful tool for optimizing bowel preparation before colonoscopy. In addition, the total Ottawa score of patients aged $<40$ years was significantly lower in the SMM group than in the control group. This may be because younger people use their smartphones more often and more 
adeptly compared to older people, which suggests that this approach using SMM may be more useful for younger patients. In our study, sending educational video clips via SMM was not helpful in improving bowel preparation in patients aged $>40$ years.

Adequate bowel preparation can be expected to increase PDR and ADR during colonoscopy. However, several recent studies reported that PDR and ADR are not closely related to the quality of bowel preparation. ${ }^{18-20}$ In our study, PDR and ADR were significantly lower in the SMM group than in the control group. Older age ( $49.9 \pm 9.6$ years vs. $46.7 \pm 9.9$ years) and longer withdrawal time during colonoscopy $(10.9 \pm 2.2$ min vs. $10.2 \pm 2.3 \mathrm{~min}$ ) in the control group than in the SMM group may have influenced these results. The incidence of severe side effects during bowel preparation including nausea/ vomiting and abdominal pain/discomfort did not significantly differ between the SMM and control groups. In addition, sleep disturbances were not significantly different between the two groups.

This study has some limitations. First, age and BMI were quite different between the two groups, although this was a randomized controlled study. The age and BMI of the patients were significantly higher in the control group than in the SMM group. These differences might have affected the bowel preparation results as well as the PDR and ADR. Second, we checked the number of views among patients in the SMM group, and they were expected to have thoroughly viewed the video clips. However, we could not confirm whether they had in fact carefully viewed the video clips, which potentially constrains the assessment of impact and the feasibility of sending video clips via SMM. Third, these results may be difficult to generalize because this study was performed in a single medical center.

In conclusion, this randomized controlled study showed that sending educational video clips via SMM improved the quality of bowel preparation, especially in the younger age group. Although further longitudinal studies will be needed to clarify the usefulness of video clips sent via SMM in optimizing bowel preparation, we expect that using a smartphone messenger service could be a helpful tool to facilitate safer and more effective colonoscopies.

\section{Conflicts of Interest}

The authors have no financial conflicts of interest.

\section{REFERENCES}

1. Sharma VK, Steinberg EN, Vasudeva R, Howden CW. Randomized, controlled study of pretreatment with magnesium citrate on the quality of colonoscopy preparation with polyethylene glycol electrolyte lavage solution. Gastrointest Endosc 1997;46:541-543.

2. Ness RM, Manam R, Hoen H, Chalasani N. Predictors of inadequate bowel preparation for colonoscopy. Am J Gastroenterol 2001;96:17971802.

3. Chung YW, Han DS, Park KH, et al. Patient factors predictive of inadequate bowel preparation using polyethylene glycol: a prospective study in Korea. J Clin Gastroenterol 2009;43:448-452.

4. Chan WK, Saravanan A, Manikam J, Goh KL, Mahadeva S. Appointment waiting times and education level influence the quality of bowel preparation in adult patients undergoing colonoscopy. BMC Gastroenterol 2011;11:86.

5. Byrne MF. The curse of poor bowel preparation for colonoscopy. Am J Gastroenterol 2002;97:1587-1590.

6. Tae JW, Lee JC, Hong SJ, et al. Impact of patient education with cartoon visual aids on the quality of bowel preparation for colonoscopy. Gastrointest Endosc 2012;76:804-811.

7. Shieh TY, Chen MJ, Chang CW, et al. Effect of physician-delivered patient education on the quality of bowel preparation for screening colonoscopy. Gastroenterol Res Pract 2013;2013:570180.

8. Liu X, Luo H, Zhang L, et al. Telephone-based re-education on the day before colonoscopy improves the quality of bowel preparation and the polyp detection rate: a prospective, colonoscopist-blinded, randomised, controlled study. Gut 2014;63:125-130.

9. Hsueh FC, Wang HC, Sun CA, et al. The effect of different patient education methods on quality of bowel cleanliness in outpatients receiving colonoscopy examination. Appl Nurs Res 2014;27:e1-e5.

10. Froehlich F, Wietlisbach V, Gonvers JJ, Burnand B, Vader JP. Impact of colonic cleansing on quality and diagnostic yield of colonoscopy: the European Panel of Appropriateness of Gastrointestinal Endoscopy European multicenter study. Gastrointest Endosc 2005;61:378-384.

11. Harewood GC, Sharma VK, de Garmo P. Impact of colonoscopy preparation quality on detection of suspected colonic neoplasia. Gastrointest Endosc 2003;58:76-79.

12. Rex DK, Imperiale TF, Latinovich DR, Bratcher LL. Impact of bowel preparation on efficiency and cost of colonoscopy. Am J Gastroenterol 2002;97:1696-1700.

13. Marmo R, Rotondano G, Riccio G, et al. Effective bowel cleansing before colonoscopy: a randomized study of split-dosage versus non-split dosage regimens of high-volume versus low-volume polyethylene glycol solutions. Gastrointest Endosc 2010;72:313-320.

14. Calderwood AH, Lai EJ, Fix OK, Jacobson BC. An endoscopist-blinded, randomized, controlled trial of a simple visual aid to improve bowel preparation for screening colonoscopy. Gastrointest Endosc 2011;73:307314.

15. Ergen WF, Pasricha T, Hubbard FJ, et al. Providing hospitalized patients with an educational booklet increases the quality of colonoscopy bowel preparation. Clin Gastroenterol Hepatol 2016;14:858-864.

16. Lee YJ, Kim ES, Choi JH, et al. Impact of reinforced education by telephone and short message service on the quality of bowel preparation: a randomized controlled study. Endoscopy 2015;47:1018-1027.

17. Cho J, Lee S, Shin JA, Kim JH, Lee HS. The impact of patient education with a smartphone application on the quality of bowel preparation for screening colonoscopy. Clin Endosc 2017;50:479-485.

18. Park JH, Kim SJ, Hyun JH, et al. Correlation between bowel preparation and the adenoma detection rate in screening colonoscopy. Ann Coloproctol 2017;33:93-98.

19. Calderwood AH, Thompson KD, Schroy PC 3rd, Lieberman DA, Jacobson BC. Good is better than excellent: bowel preparation quality and adenoma detection rates. Gastrointest Endosc 2015;81:691-699.el.

20. Adike A, Buras MR, Gurudu SR, et al. Is the level of cleanliness using segmental Boston bowel preparation scale associated with a higher adenoma detection rate? Ann Gastroenterol 2018;31:217-223. 